\title{
ICHTHYOFAUNAL DIVERSITY OF RANJIT SAGAR WETLAND SITUATED IN THE NORTHWESTERN HIMALAYAS
}

\section{ONKAR SINGH BRRAICH ${ }^{1, *}$ and SUNIL KUMAR SAINI ${ }^{2}$}

\author{
${ }^{1}$ Department of Zoology and Environmental Sciences, Punjabi University, Patiala, Punjab, India \\ 2 Department of Biotechnology and Medical Sciences, Baba Farid College, Deon, Bathinda, Punjab, India \\ * Corresponding author: singhonkar@yahoo.com
}

\begin{abstract}
Diversity of fishes was evaluated at the Ranjit Sager Wetland and its three adjoining streams. Factors like altitude, bed gradient, dominated substratum and habitat types of the streams have also been studied. These streams fall under Type-B category on the basis of habitat variability, gradient and sinuosity. 43 fish species represented by 6 orders have been reported from the study area. Out of all 43 fish species, 13 come under threatened categories of Red List of IUCN, out of which 2, 3 and 8 come under Endangered (EN), Vulnerable (VU) and Low Risk near threatened category (LRnt) respectively. It has been analyzed from the above pattern that maximum fish species reported from this wetland have fallen under different threatened categories.
\end{abstract}

Keywords: abundance; habitat loss; species richness; threatened species

\section{Introduction}

The heterogeneous freshwater habitat in rivers, streams, springs and headwaters like variation in altitude, flow rates, physical substrate and the riparian zones provides good opportunity of food, shade and cover for various fish species. Consequently, freshwater habitats harbor diverse fauna, with fish serving as prime indicators of ecosystem status (Armantrout 1990). Though study of assemblage pattern and partitioning of fish diversity is a challenging subject in fishery science (Ross 1986). Fish research has become an increasingly important study area, as fish population is declining throughout the world due to various anthropogenic activities. The decline of fishes has an adverse impact on aquatic ecosystems as well as a significant impact on human population as it is one of the primary food sources for human. Anthropogenic activities such as modification of the environment, culture, harvesting and effects of modernization have contributed to the pollution of water bodies which serve as habitat for fishes (Plafkin et al. 1989; Siligato and Bohmer 2001; Vijaylaxmi et al. 2010; Tiwari 2011). Due to rise of population in all countries, fishes may play an important role in economic development of countries (Sikoki and Otobotekere 1999; Gharaei et al. 2010; 2011).

In India it is estimated that about 2500 fish species are found within which around 930 species are freshwater. The freshwater fishes are distributed amongst approximately 20 orders, 100 families and 300 genera (Daniels 2000; Kar 2003; Ayappan and Birdar 2004). Fishes are the main component of lake and wetlands ecosystems. They play an important role in energy flows, nutrient cycling and maintaining community balances in these ecosystems. The physical, chemical and biological characteristics of a wetland are major determinants of the type, number and size of fish available (Baker et al. 1993; Abbasi 1997).

Fish biodiversity was studied in Punjab and described as many as 116 fish species (Johal and Tandon 1979; 1980). Dua and Chander (1999) have identified 61 species from Harike wetland. Brraich et al. (2003) identified 3 new fish species from Harike wetland viz., Nandus nandus, Lepidocephalichthys guntea and Monopterus chuchia. Further, Brraich and Ladhar (2005) identified one more species viz., Badis badis. Furthermore, they also reported 69 fish species from Harike, 55 from Ropar and 16 from Kanjli wetland.

There are various environmental factors which affect fish communities in freshwaters. The most important are streamflow, water quality, food sources, physical habitat and biotic interactions that affect stream fish and aquatic communities. At the most general level of resolution, channel units are divided into fast and slow-water categories that approximately correspond to the commonly used terms "riffle" and "pool". Within the fast - water category, two subcategories of habitats are identified, those that are highly turbulent (falls, cascade, chutes, rapids and riffles) and those with low turbulence (sheets and runs). Different habitat harbour different verities of fish species (Hawkins et al. 1993).

Ranjit Sagar Wetland and its adjoining streams are one of the great potential (Johal et al. 2002) fishery resources in India. The research on fish species of this wetland has not been conducted earlier. The detailed study on fish diversity observed from this wetland will extend great help to start conservation and management practices in future. 


\section{Materials and Methods}

The Ranjit Sagar wetland, also known as Thein Dam, is a fresh water ecosystem situated on the river Ravi (tributary of the Indus river system) near Pathankot city, Punjab, India. This wetland falls into three states i.e. Punjab, Himachal Pradesh and Jammu and Kashmir and spread over an area of $87.60 \mathrm{sq} \mathrm{km}$ and catchment area consist of $6086 \mathrm{sq} \mathrm{km}$. There are three major streams (Karnal, Basoli and Behni) feeding Ranjit Sagar Wetland (Fig. 1, Table 1). Fish samples were collected on monthly basis from each stream by selecting three fish collection sites with difference of $1 \mathrm{~km}$ each. The fish sample were collected in triplicate with the help of local fishermen by using standard fishing gears like cast, gill and hand nets. The sampling was made from different habitats such as riffles, cascades, ripples and runs (Rosgen 1996). After collection, fish specimen were examined, counted and released into the water. The unidentified specimen were preserved in 5\% formalin and brought to the laboratory for further analysis and identification. Fish specimens were identified on the basis of morphological characters and with the help of standard keys and taxonomy text books (Johal and Tandon 1979; Day 1888; Jayaram 2010). Rosgen (1996) classified the hillstreams into three major types Type A, B and F. Type-A streams is high altitude streams with high gradient. These streams have stable bed morphology with boulders or bedrock dominated channels. Type-B streams are with gentle gradient, cobble gravel dominated substrate with variable habitat types. Type-F streams are similar to Type-B except that they are more entrenched in the highly weathered materials.

Table 1 List of study sites of Ranjit Sagar Wetland.

\begin{tabular}{|c|l|l|c|c|c|l|l|}
\hline $\begin{array}{c}\text { Sr. } \\
\text { No. }\end{array}$ & \multicolumn{1}{|c|}{ Site } & Location & $\begin{array}{c}\text { Altitude } \\
(\mathbf{m})\end{array}$ & Latitude & Longitude & Substrate \\
\hline 1. & $\begin{array}{l}\text { Dam } \\
\text { (Reservoir) }\end{array}$ & Punjab & 1697 & $75^{\circ} 45^{\prime} 45^{\prime \prime} \mathrm{E}$ & $32^{\circ} 26^{\prime} 37^{\prime \prime} \mathrm{N}$ & Rocky & Deep pools and shallow pools \\
\hline 2. & $\begin{array}{l}\text { Behni } \\
\text { (Stream) }\end{array}$ & $\begin{array}{l}\text { Jammu and } \\
\text { Kashmir }\end{array}$ & 1778 & $75^{\circ} 39^{\prime} 10.88^{\prime \prime} \mathrm{E}$ & $32^{\circ} 31^{\prime} 39.79^{\prime \prime} \mathrm{N}$ & $\begin{array}{l}\text { mostly bedrock, boulders, bravel, } \\
\text { cobble and leaf litter }\end{array}$ & Pools, riffles, cascade, run \\
\hline 3. & $\begin{array}{l}\text { Basoli } \\
\text { (Stream) }\end{array}$ & $\begin{array}{l}\text { Himachal } \\
\text { Pradesh }\end{array}$ & 1768 & $75^{\circ} 50^{\prime} 46.30^{\prime \prime} \mathrm{E}$ & $32^{\circ} 30^{\prime} 35.95^{\prime \prime} \mathrm{N}$ & $\begin{array}{l}\text { Boulders, gravel, cobble } \\
\text { and sand }\end{array}$ & $\begin{array}{l}\text { Deep pools, pools, riffles and } \\
\text { cascade }\end{array}$ \\
\hline 4. & $\begin{array}{l}\text { Karnal } \\
\text { (stream) }\end{array}$ & Punjab & 2118 & $75^{\circ} 53^{\prime} 01.53^{\prime \prime} \mathrm{E}$ & $32^{\circ} 27^{\prime} 01.27^{\prime \prime} \mathrm{N}$ & $\begin{array}{l}\text { Mix with big boulders, gravel, } \\
\text { cobble and leaf litter }\end{array}$ & Pools, riffles, cascade and run \\
\hline
\end{tabular}

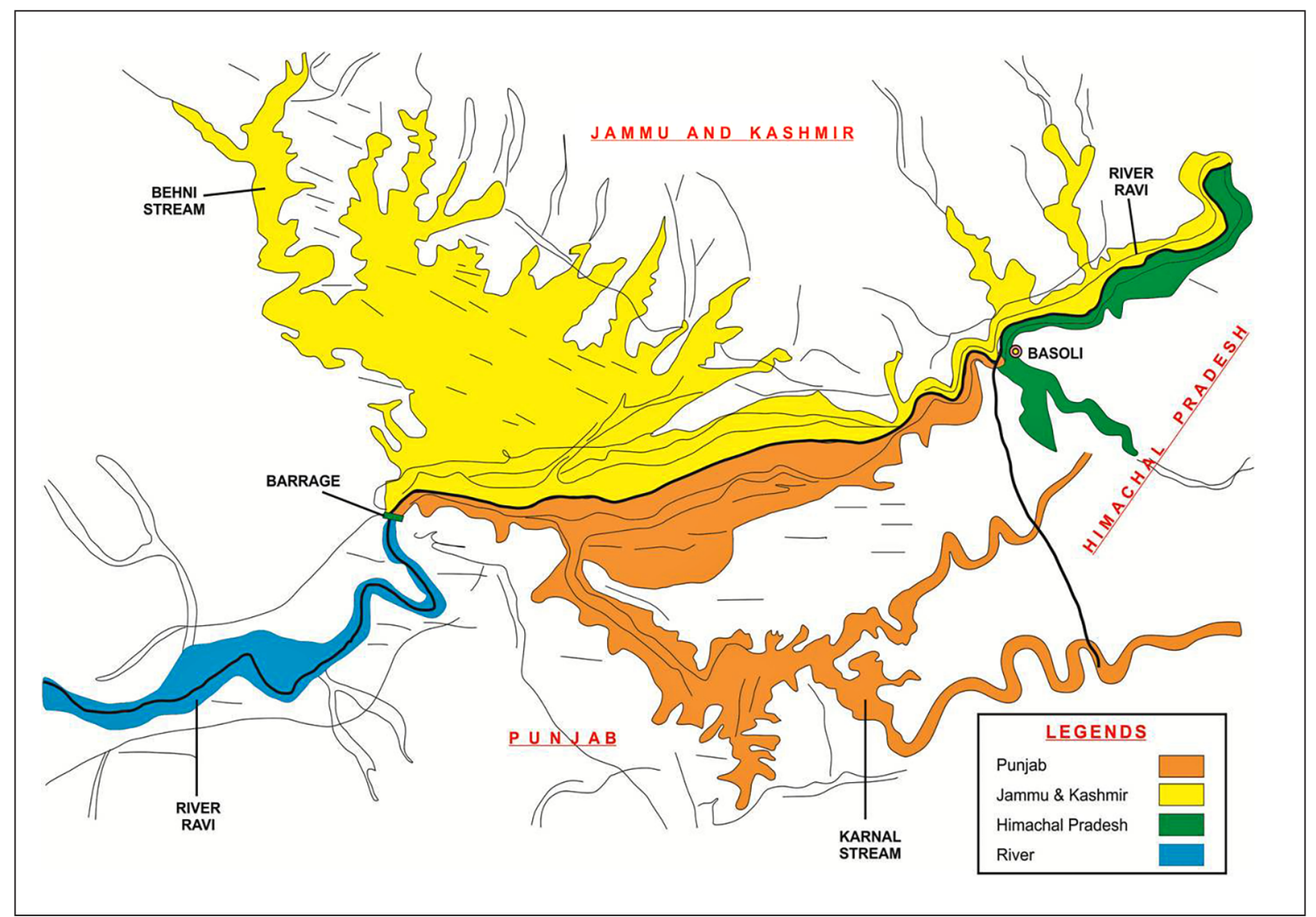

Fig. 1 Map of Ranjit Sagar Wetland. 


\section{Results}

A total of 43 fish species have been reported from the Ranjit Sagar Wetland and its adjoining streams belonging to 6 orders, 11 families and 27 genera (Table 2). Cyprinidae forms the dominant group among the families with $64 \%$ followed by Channidae with $9 \%$, Cobitidae with $5 \%$, Siluridae with $5 \%$, Balitoridae with $5 \%$, and other fami-

Table 2 Classification of the fish species reported from the Ranjit Sagar Wetland and adjoining streams.

\begin{tabular}{|c|c|c|c|}
\hline S. No. & Name of Fish & English Name & Habitat Preference \\
\hline $\mathbf{I}$ & \multicolumn{3}{|l|}{ Order - Cypriniformes } \\
\hline \multirow[t]{29}{*}{ i } & Family - Cyprinidae & & \\
\hline & Bangana dero & Kalabans & $\begin{array}{l}\text { Inhabit hill-streams in shallow waters. Adults migrate to warmer regions } \\
\text { of lakes and streams during winter. }\end{array}$ \\
\hline & Barilius bendelisis & Hamilton's Barila & $\begin{array}{l}\text { Base of hills in the lotic habitat strewned with pebbles and sandy } \\
\text { bottom. }\end{array}$ \\
\hline & Barilius shacra & Shacra Baril & Found in streams and rivers. \\
\hline & Barilius vagra & Vagra Barila & Found in hill streams with gravelly and rocky bottom. \\
\hline & Cabdio morar & Aspidoparia & Found in streams, rivers and ponds in plains and mountainous regions \\
\hline & Cirrhinus reba & Reba Carp & Found in large streams, rivers, tanks, lakes and reservoirs \\
\hline & Devario devario & Devario danio & Fast flowing clear headwater streams. \\
\hline & Crossocheilus latius latius & Gangetic Latia & $\begin{array}{l}\text { Inhabits streams, rivers and lake preferably with gravelly bottom in } \\
\text { benthopelagic environment. }\end{array}$ \\
\hline & Ctenopharyngodon idellus & Grass Carp & $\begin{array}{l}\text { Inhabit large rivers, lakes, and reservoirs with abundant vegetation and } \\
\text { relatively shallow waters. }\end{array}$ \\
\hline & Cyprinus carpio & Common Carp & $\begin{array}{l}\text { Warm, deep, slow-flowing and still waters, such as lowland rivers and } \\
\text { large, well vegetated lakes. }\end{array}$ \\
\hline & Garra gotyla gotyla & Sucker Head & $\begin{array}{l}\text { This species is found in fast flowing streams with boulders and rocks } \\
\text { along the Himalayan ranges. }\end{array}$ \\
\hline & Hypophthalamichthys molitrix & Silver Carp & $\begin{array}{l}\text { It migrates upstream to breed; egg and larva float downstream to } \\
\text { floodplain zones. }\end{array}$ \\
\hline & Hypophthalamichthys nobilis & Big Head & $\begin{array}{l}\text { Inhabits rivers with marked water-level fluctuations, overwinters in } \\
\text { middle and lower stretches. }\end{array}$ \\
\hline & Labeo bata & Bata Labeo & Found in streams and rivers. \\
\hline & Labeo dyocheilus & Brahmaputra Labeo & Inhabits clear active currents of large rivers \\
\hline & Labeo gonius & Kuria Labeo & It inhabits rivers and streams. \\
\hline & Labeo pangusia & Pangusia Labeo & It inhabits mountain streams, rivers, lakes and ponds \\
\hline & Labeo rohita & Rohu & It inhabits rivers and streams. \\
\hline & Pethia conchonius & Rosy Barb, Red barb & Generally inhabits lakes and streams. \\
\hline & Pethia phutunio & $\begin{array}{l}\text { Spotted Tail Barb, Pygmy } \\
\text { Barb, Dwarf Barb }\end{array}$ & Inhibits clear streams and rivers, also muddy waters \\
\hline & Pethia ticto & $\begin{array}{l}\text { Ticto Barb, Firefin Barb, } \\
\text { Two-Spot Barb }\end{array}$ & Inhabits mostly mountain and sub-mountain regions, and flood plains. \\
\hline & Puntius chola & Swamp Barb, Chola Barb & It inhabits rivers, streams and tanks in the plains \\
\hline & Rasbora daniconius & $\begin{array}{l}\text { Slender Rasbora, Bliack line } \\
\text { Rasbora }\end{array}$ & $\begin{array}{l}\text { It occurs in a variety of habitats: ditches, ponds, canals, streams, rivers } \\
\text { and inundated fields, but is primarily found in sandy streams and rivers. }\end{array}$ \\
\hline & Salmophasia bacaila & Large Razorbelly Minnow & $\begin{array}{l}\text { Usually found in slow running streams, but also occurring in rivers, } \\
\text { ponds and inundated fields in sub-mountain regions }\end{array}$ \\
\hline & Systomus sarana sarana & Olive Barb & It can live in sandy bed mixed with mud and in fairly swift current. \\
\hline & Tor chylenoides & Dark Mahseer & Inhabits fast-flowing mountain streams \\
\hline & Tor putitora & $\begin{array}{l}\text { Golden Mahaseer, Putitor } \\
\text { Mahseer }\end{array}$ & It inhabits rapid streams with rocky bottom, riverine pools and lakes. \\
\hline & Tortor & Tor Mahseer & It grows better in rivers with a rocky bottom. \\
\hline \multirow[t]{2}{*}{ ii } & \multicolumn{3}{|l|}{ Family - Balitoridae } \\
\hline & Acanthocobitis botia & & $\begin{array}{l}\text { Inhabits swift flowing streams in hilly areas with clear water and rocky, } \\
\text { pebbly and sandy bottoms. }\end{array}$ \\
\hline
\end{tabular}




\begin{tabular}{|c|c|c|c|}
\hline \multirow[t]{2}{*}{ iii } & \multicolumn{3}{|l|}{ Family-Cobitidae } \\
\hline & Botia birdi & Botia Loach & Occurs in clear mountain streams. \\
\hline & Botia lohachata & Y-Loach, Reticulate Loach & Occurs in clear mountain streams. \\
\hline II & \multicolumn{3}{|l|}{ Order - Siluriformes } \\
\hline \multirow[t]{2}{*}{ i } & \multicolumn{3}{|l|}{ Family - Clariidae } \\
\hline & Heteropneustes fossilis & Stinging Catfish & $\begin{array}{l}\text { Inhabits freshwater, rarely brackish waters. This is primarily a fish of } \\
\text { ponds, ditches, bheels, swamps and marshes, but it is sometimes found } \\
\text { in muddy rivers. }\end{array}$ \\
\hline \multirow[t]{3}{*}{ ii } & \multicolumn{3}{|l|}{ Family - Siluridae } \\
\hline & Ompok bimaculatus & Indian Butter Catfish & $\begin{array}{l}\text { Inhabits plains and sub-mountain regions, and is found in rivers, lakes, } \\
\text { tanks and ponds. }\end{array}$ \\
\hline & Ompokpabda & Pabdah Catfish & The species inhabits lotic habitats such as rivers and larger streams. \\
\hline \multirow[t]{2}{*}{ iii } & \multicolumn{3}{|l|}{ Family - Sisoridae } \\
\hline & Glyptothorax punjabensis & & $\begin{array}{l}\text { The species inhabits benthopelagic zones of lotic habitats such as rivers } \\
\text { and larger streams. }\end{array}$ \\
\hline III & \multicolumn{3}{|l|}{ Order - Synbranchiiformes } \\
\hline \multirow[t]{2}{*}{$\mathrm{i}$} & Family - Synbranchidae & & \\
\hline & Mastacembelus armatus & Tire-Track Spinyeel & Inhabits fresh waters in plains and hills. \\
\hline IV & \multicolumn{3}{|l|}{ Order- Beloniformes } \\
\hline \multirow[t]{2}{*}{ i } & \multicolumn{3}{|l|}{ Family-Belonidae } \\
\hline & Xenentodon cancila & Freshwater Garfish & $\begin{array}{l}\text { Inhabits freshwaters, primarily rivers. It occurs in clear, gravelly, perenni- } \\
\text { al streams and ponds. }\end{array}$ \\
\hline $\mathbf{v}$ & \multicolumn{3}{|l|}{ Order-Perciformes } \\
\hline \multirow[t]{5}{*}{$\mathrm{i}$} & \multicolumn{3}{|l|}{ Family - Channidae } \\
\hline & Channa marulius & Giant Snakehead & $\begin{array}{l}\text { Inhabits large lakes and rivers; prefers deep, clear stretches of water } \\
\text { with sandy or rocky bottoms. }\end{array}$ \\
\hline & Channa orientalis & Asiatic Snakehead & $\begin{array}{l}\text { Species occurring in rivers, lakes, ponds, mountain streams and even } \\
\text { brackish water. Found in quiet, shaded, clear, flowing water with silt or } \\
\text { gravel substrate. }\end{array}$ \\
\hline & Channa punctatus & Spotted Snakehead & $\begin{array}{l}\text { Inhabits freshwater streams, rivers, ponds and tanks, generally in the } \\
\text { plains. Also found in rice fields and irrigation channels. }\end{array}$ \\
\hline & Channa striata & $\begin{array}{l}\text { Striped or Banded Snake- } \\
\text { head }\end{array}$ & $\begin{array}{l}\text { Inhabits swamps, freshwater ponds, streams and tanks in the plains; } \\
\text { prefers stagnant muddy waters and grassy tanks. }\end{array}$ \\
\hline \multirow[t]{2}{*}{ ii } & \multicolumn{3}{|l|}{ Family - Nandidae } \\
\hline & Nandus nandus & Mottled Nandus & It inhabits fresh waters. Found in rivers and in agricultural lands. \\
\hline VI & Order-Osteoglossiformes & & \\
\hline \multirow[t]{2}{*}{$\mathrm{i}$} & Family - Notopteridae & & \\
\hline & Notopterus notopterus & Grey Featherback & $\begin{array}{l}\text { It inhabits fresh and brackish waters, and appears to thrive well in lentic } \\
\text { waters. }\end{array}$ \\
\hline
\end{tabular}

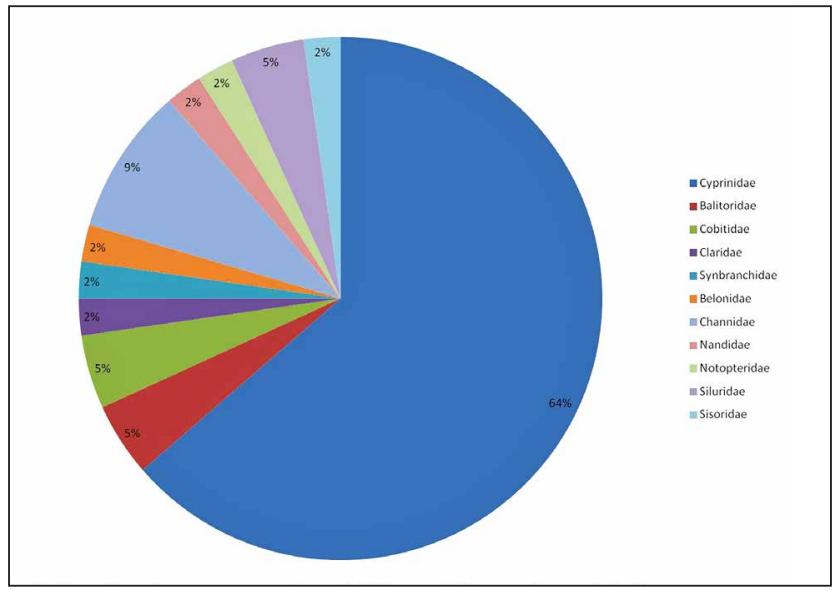

Fig. 2 Family-wise representation of different fish species and their percentage.

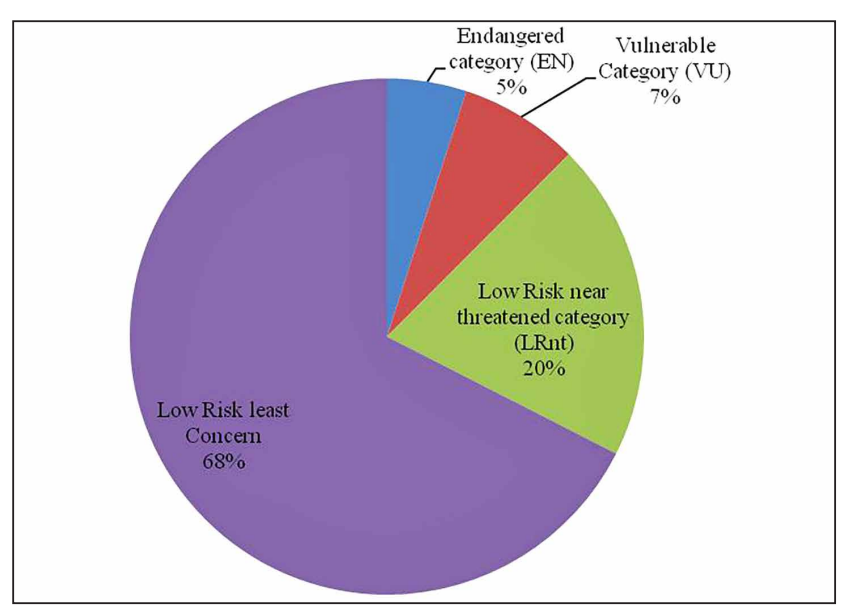

Fig. 3 Percentage-wise IUCN Red List status. 
Table 3 Status of fishes from Ranjit Sagar Wetland and adjoining streams based on Red Data list of IUCN (2017) and criteria, threats \& research recommendations according to CAMP Report (Molur and Walker 1998).

\begin{tabular}{|c|c|c|c|c|c|c|}
\hline S. No. & Name of Fish & $\begin{array}{l}\text { IUCN } \\
\text { Status }\end{array}$ & Criteria & Sites & Threats & $\begin{array}{c}\text { Research } \\
\text { Recommended }\end{array}$ \\
\hline 1. & Acanthocobitis botia & LRIC & - & $\mathrm{Ba}, \mathrm{Be}, \mathrm{K}$ & $\mathrm{Fd}, \mathrm{E}, \mathrm{F}, \mathrm{I}, \mathrm{L}, \mathrm{Po}, \mathrm{Ov}, \mathrm{Sn}, \mathrm{T}(\mathrm{L})$ & Lh, Hm, M, PP \\
\hline 2. & Bangana dero & LRIC & (A1acd) & $\mathrm{Ba}, \mathrm{Be}, \mathrm{D}, \mathrm{K}$ & $\mathrm{Dm}, \mathrm{Dr}, \mathrm{Fd}, \mathrm{F}, \mathrm{L}, \mathrm{H}, \mathrm{I}, \mathrm{Lp}, \mathrm{Ov}, \mathrm{Sn}, \mathrm{T}(\mathrm{L}, \mathrm{D})$ & $\mathrm{S}, \mathrm{M}, \mathrm{Hm}, \mathrm{Lm}$ \\
\hline 3. & Barilius bendelisis & LRIC & - & $\mathrm{Ba}, \mathrm{Be}, \mathrm{D}, \mathrm{K}$ & $\mathrm{Fd}, \mathrm{F}, \mathrm{I}, \mathrm{L}, \mathrm{Ov}, \mathrm{Po}, \mathrm{Sn}, \mathrm{T}(\mathrm{L}, \mathrm{C})$ & $\mathrm{M}, \mathrm{Lh}, \mathrm{Hm}, \mathrm{P}$ \\
\hline 4. & Barilius shacra & LRnt & - & $\mathrm{Ba}, \mathrm{Be}, \mathrm{D}, \mathrm{K}$ & I, L, Pu, Sn, T(L) & $\mathrm{M}, \mathrm{O}, \mathrm{P}$ \\
\hline 5. & Barilius vagra & LRIC & $(\mathrm{A} 1 \mathrm{a}, 1 \mathrm{c})$ & $\mathrm{Ba}, \mathrm{Be}, \mathrm{D}, \mathrm{K}$ & $\mathrm{I}, \mathrm{L}, \mathrm{T}(\mathrm{L})$ & $\mathrm{S}, \mathrm{M}, \mathrm{Hm}, \mathrm{P}$ \\
\hline 6. & Botia birdi & LRnt & - & $\mathrm{Be}$ & I, L, Pu, Sn, F, T(L) & $\mathrm{Hm}, \mathrm{Lh}, \mathrm{O}, \mathrm{P}$ \\
\hline 7. & Botia lohachata & EN & $(\mathrm{B} 1,2 \mathrm{C})$ & $\mathrm{Be}$ & $\mathrm{Fd}, \mathrm{E}, \mathrm{I}, \mathrm{L}, \mathrm{Ov}, \mathrm{Po}, \mathrm{Sn}, \mathrm{T}(\mathrm{L})$ & Lh, Hm, M, P \\
\hline 8. & Cabdio morar & LRIC & - & $\mathrm{Be}$ & $\mathrm{I}, \mathrm{L}, \mathrm{Ov}, \mathrm{Pu}, \mathrm{T}(\mathrm{L}, \mathrm{C})$ & $S, M, L h, T, G$ \\
\hline 9. & Channa marulius & LRIC & - & $\mathrm{Ba}, \mathrm{Be}, \mathrm{K}$ & $F, L, O v, T(D, C)$ & $M, H$ \\
\hline 10. & Channa orientalis & Vu & (A1acd) & $\mathrm{Be}, \mathrm{K}$ & $F, L, T(D)$ & $\mathrm{Hm}, \mathrm{S}, \mathrm{M}, \mathrm{PP}$ \\
\hline 11. & Channa punctatus & LRIC & - & $\mathrm{Ba}, \mathrm{Be}, \mathrm{D}$ & $F, L, O v, T(D)$ & $\mathrm{H}, \mathrm{Hm}$ \\
\hline 12. & Channa striata & LRIC & & D & $\mathrm{F}, \mathrm{T},(\mathrm{C})$ & $\mathrm{H}$ \\
\hline 13. & Cirrhinus reba & LRIC & (A1abcd, 2cd) & $\mathrm{Ba}, \mathrm{Be}, \mathrm{D}, \mathrm{K}$ & Dm, F, I, L, Ov, Pu, Sn, T(D,C) & S, M \\
\hline 14. & Crossocheilus latius latius & LRIC & - & $\mathrm{Ba}, \mathrm{Be}, \mathrm{K}$ & $\mathrm{L}, \mathrm{Fd}$ & Lh \\
\hline 15. & Ctenopharyngodon idellus & DD & - & $\mathrm{Ba}, \mathrm{D}, \mathrm{K}$ & - & - \\
\hline 16. & Cyprinus carpio & Vu & (A2ce) & $\mathrm{Ba}, \mathrm{D}, \mathrm{K}$ & - & - \\
\hline 17. & Devario devario & LRnt & - & $\mathrm{Be}, \mathrm{K}$ & $\mathrm{I}, \mathrm{Ov}, \mathrm{Po}, \mathrm{Pu}, \mathrm{T}(\mathrm{L}, \mathrm{D})$ & $\begin{array}{l}\text { S, M, Lr, Hm, } \\
\text { Lh, P }\end{array}$ \\
\hline 18. & Garra gotyla gotyla & LRIC & (A1acd) & $\mathrm{Ba}, \mathrm{Be}, \mathrm{K}$ & $\mathrm{Fd}, \mathrm{E}, \mathrm{I}, \mathrm{L}, \mathrm{Ov}, \mathrm{Ps}, \mathrm{Po}, \mathrm{Sn}, \mathrm{T}(\mathrm{L})$ & $\mathrm{M}, \mathrm{Lh}, \mathrm{Hm}, \mathrm{P}$ \\
\hline 19. & Glyptothorax punjabensis & DD & - & $\mathrm{Be}, \mathrm{K}$ & - & - \\
\hline 20. & Heteropneustes fossilis & LRIC & (A1acd) & $\mathrm{Ba}, \mathrm{K}$ & $F, I, L, T(L, D, C)$ & $\mathrm{S}, \mathrm{M}, \mathrm{H}, \mathrm{Hm}, \mathrm{P}$ \\
\hline 21. & Hypophthalamichthys molitrix & LRnt & - & $\mathrm{Ba}, \mathrm{D}$, & - & - \\
\hline 22. & Hypophthalamichthys nobilis & DD & - & $\mathrm{Ba}, \mathrm{D}$ & - & - \\
\hline 23. & Labeo bata & LRIC & - & $\mathrm{Be}, \mathrm{D}, \mathrm{K}$ & L, Ov, Sn, T (C) & M \\
\hline 24. & Labeo dyocheilus & LRIC & (A1acd) & $\mathrm{Ba}, \mathrm{D}$ & Dm, Dr, Fd, F, I, H, L, Ov, T(L,D) & $\begin{array}{l}\mathrm{S}, \mathrm{M}, \mathrm{Hm}, \mathrm{Lm}, \\
\mathrm{PP}\end{array}$ \\
\hline 25. & Labeo gonius & LRIC & - & $\mathrm{Be}, \mathrm{K}$ & $\mathrm{I}, \mathrm{H}, \mathrm{Ov}, \mathrm{Pu}, \mathrm{T}(\mathrm{C})$ & $G, M, S$ \\
\hline 26. & Labeo pangusia & LRnt & - & $\mathrm{Be}, \mathrm{K}$ & $\mathrm{I}, \mathrm{H}, \mathrm{Ov}, \mathrm{Pu}, \mathrm{T}(\mathrm{D})$ & $\mathrm{S}, \mathrm{M}, \mathrm{Lh}, \mathrm{Hm}$ \\
\hline 27. & Labeo rohita & LRIC & - & $\mathrm{Ba}, \mathrm{Be}, \mathrm{K}$ & $L, O v, S n, T(C)$ & G \\
\hline 28. & Mastacembelus armatus & LRIC & - & $\mathrm{Ba}, \mathrm{Be}, \mathrm{D}, \mathrm{K}$ & - & - \\
\hline 29. & Nandus nandus & LRIC & - & $\mathrm{Ba}, \mathrm{Be}, \mathrm{K}$ & I, L, Po, Pu, Sn, T(L) & $S, M, G, P$ \\
\hline 30. & Notopterus notopterus & LRIC & - & $\mathrm{Ba}, \mathrm{D}, \mathrm{K}$ & $\mathrm{I}, \mathrm{Ov}, \mathrm{Pu}, \mathrm{T}(\mathrm{C})$ & M \\
\hline 31. & Ompok bimaculatus & LRnt & $(\mathrm{A} 1 \mathrm{acd}, 2 \mathrm{~cd})$ & $\mathrm{Ba}, \mathrm{D}, \mathrm{K}$ & $\mathrm{D}, \mathrm{Fd}, \mathrm{F}, \mathrm{I}, \mathrm{Po}, \mathrm{Ps}, \mathrm{Pu}, \mathrm{Sn}, \mathrm{T}(\mathrm{L}, \mathrm{D}, \mathrm{C})$ & $\begin{array}{l}\mathrm{S}, \mathrm{M}, \mathrm{G}, \mathrm{Hm}, \\
\mathrm{Lm}, \mathrm{P}\end{array}$ \\
\hline 32. & Ompokpabda & LRnt & $(\mathrm{A} 1 \mathrm{acd}, 2 \mathrm{~cd})$ & $\mathrm{Ba}, \mathrm{D}, \mathrm{K}$ & $\mathrm{F}, \mathrm{I}, \mathrm{Pu}, \mathrm{T}(\mathrm{L}, \mathrm{D}, \mathrm{C})$ & $\begin{array}{l}\mathrm{S}, \mathrm{M}, \mathrm{G}, \mathrm{Hm}, \mathrm{Lm}, \\
\mathrm{Lr}, \mathrm{P}\end{array}$ \\
\hline 33. & Pethia conchonius & LRIC & $\mathrm{B} 1,2 \mathrm{C}$ & $\mathrm{Ba}, \mathrm{Be}, \mathrm{D}, \mathrm{K}$ & $E, L, P o, S n, T(L)$ & Lh, Hm, M \\
\hline 34. & Pethia phutunio & LRIC & - & $\mathrm{Ba}, \mathrm{Be}, \mathrm{K}$ & $\mathrm{T}(\mathrm{C})$ & $\mathrm{S}, \mathrm{M}$ \\
\hline 35 & Pethia ticto & LRIC & - & $\mathrm{Ba}, \mathrm{Be}, \mathrm{D}$ & $F, L, T(L)$ & $\mathrm{Hm}$ \\
\hline 36. & Puntius chola & LRIC & $\mathrm{A} 1 \mathrm{a}, 1 \mathrm{c}, 1 \mathrm{~d}$ & $\mathrm{Be}, \mathrm{D}, \mathrm{K}$ & $\mathrm{I}, \mathrm{Pu}, \mathrm{T}(\mathrm{L})$ & $\mathrm{S}, \mathrm{M}$ \\
\hline 37. & Rasbora daniconius & LRIC & - & $\mathrm{Be}, \mathrm{K}$ & $\mathrm{F}, \mathrm{Pu}, \mathrm{T}(\mathrm{L}, \mathrm{D})$ & $\mathrm{S}, \mathrm{M}, \mathrm{G}, \mathrm{Hm}$ \\
\hline 38. & Salmophasia bacaila & LRIC & - & K & $\mathrm{T}(\mathrm{L})$ & Lh, M, S \\
\hline 39. & Systomus sarana sarana & LRIC & A1acd & $\mathrm{Ba}, \mathrm{Be}, \mathrm{D}, \mathrm{K}$ & $F, I, L, T(L, D)$ & $\mathrm{S}, \mathrm{M}, \mathrm{Lr}, \mathrm{P}$ \\
\hline 40. & Tor chylenoides & Vu & - & $\mathrm{Ba}, \mathrm{Be}, \mathrm{D}, \mathrm{K}$ & - & - \\
\hline 41. & Tor putitora & EN & A1acd & $\mathrm{Ba}, \mathrm{Be}, \mathrm{D}, \mathrm{K}$ & $\mathrm{Dm}, \mathrm{Dr}, \mathrm{Fd}, \mathrm{F}, \mathrm{I}, \mathrm{H}, \mathrm{L}, \mathrm{Ov}, \mathrm{Pi}, \mathrm{Sn}, \mathrm{T}(\mathrm{L}, \mathrm{D})$ & $\mathrm{S}, \mathrm{M}, \mathrm{Hm}, \mathrm{Lm}, \mathrm{P}$ \\
\hline 42. & Tor tor & LRnt & $\mathrm{A} 1 \mathrm{a}, 1 \mathrm{c}, 1 \mathrm{~d}$ & $\mathrm{Be}, \mathrm{D}$ & $\mathrm{Dm}, \mathrm{Fd}, \mathrm{F}, \mathrm{I}, \mathrm{L}, \mathrm{Po}, \mathrm{Pu}, \mathrm{T}(\mathrm{L}, \mathrm{D}, \mathrm{C})$ & $\begin{array}{l}\mathrm{S}, \mathrm{M}, \mathrm{G}, \mathrm{Hm}, \mathrm{Lm}, \\
\mathrm{Lr}, \mathrm{P}\end{array}$ \\
\hline 43. & Xenentodon cancila & LRIC & - & $\mathrm{Ba}, \mathrm{Be}, \mathrm{D}, \mathrm{K}$ & $\mathrm{F}, \mathrm{Pu}, \mathrm{T}(\mathrm{D})$ & $S, M, L r, P$ \\
\hline
\end{tabular}




\section{Abbreviations}

Site: Be - Behni, Ba - Basoli, K - Karnal, D - Dam.

IUCN: EN - Endangered, VU - Vulnerable, LRnt - Lower Risk-near threatened, LRIc - Lower Risk-near least concern.

Threats: I - Human interference, L - Loss of habitat, Lf - Loss of habitat due to fragmentation, Lp - Loss of habitat due to exotic plants, D - Diseases, Dm - Damming, E - Edaphic factors, F - Fishing, Fd - Destructive fishing, H - Harvest, Hf - Harvest for food, P - Predation, Po - Poisoning, Ps Pesticides, Pu - Pollution, Pi - Powerlines, $\mathrm{Sn}$ - Siltation, T - Trade , Ov - Overexploitation, Dr - Drowning.

Research Recommendations: S - Survey search and find, M - Monitoring, $\mathrm{H}$ - Husbandry research, $\mathrm{Hm}$ - Habitat management, Lr - Limiting factor research, Lm - Limiting factor management, Lh - Life history studies, T - Taxonomic and Morphological genetics studies, G - Genetic Managements, P - Population and habitat viability assessment, PP - PHVA (Pending Further Work), O - Other (Specific to the Species).

lies namely Synbranchidae, Belonidae, Claridae, Nandinae and Notopteridae forming 2\% each (Fig. 2). Out of all 43 fish species, 13 come under threatened categories of Red List of IUCN (Table 3), out of which 2, 3 and 8 come under Endangered (EN), Vulnerable (VU) and Low Risk near threatened category (LRnt) respectively (Fig. 3). This wetland also supports four exotic fish species i.e. Cyprinus carpio, Ctenopharyngodon idellus, Hypophthalmichthys molitrix and H. nobilis. During the study period different type of streams habitats have been studied. All three streams Behni, Basoli and Karnal were dominated by different type of habitats like deep pools, pools, riffles, runs and cascade. Streams banks were stable and covered by riparian vegetation. Different types of stream substrates have also been studied during the course of work in which bedrock type of substrate was predominant, other type of substrates viz. big boulders, gravel, cobble, sand and leaf litter was also present. These types of streams were called Type-B streams which are very productive in nature and support large variety of fish diversity.

\section{Discussion}

During the course of study, 25 fish species have been reported from Dam site, 29 from Basoli and 33 each from Behni and Karnal site. A total of 43 fish species have been reported from the Ranjit Sagar Wetland and its adjoining streams were classified under different order and families. Goswami and Goswami (2006) have identified 54 fish species belonging to 36 genera under 22 families in Jamalai wetland in Assam. Sharma et al. (2007) reported 29 species of fishes belonging to six orders from $\mathrm{Kr}$ ishnapura lake, Indore and stated that Cypriniformes was dominant with 15 species followed by Siluriformes with 6 species. Jagatheeswari et al. (2016) also studied the diversity of fish population and their conservation aspects in Kondakarla fresh water lake ecosystem, Visakhapatnam, Andhra Pradesh, India and reported 26 species of fishes.

Occurrence of variety of fish species depends upon the availability of different habitats (Arunachalam 2000). Hence, fishes have also been classified on the basis of their habitat preferences. The dominant fish habitats in the streams were cascade, rapids, riffles and run. Similarly, dam has deep pools in the middle and shallow pools near the banks. Rosenzweib (1995) revealed that number of species increase with an increase in habitat area which supports the phenomenon of species habitat area relationship. Arunachalam (2000) studied the macro and microhabitat of 10 streams of Western Ghats and provide information about the habitat requirement of fish species in different stream/rivers. They stated that habitat diversity is directly related to fish diversity.

Kar and Sen (2007) studied the distribution of fishes on the basis of habitat preference. During the study we study that, the edges of the run habitats have been found to be inhabited mainly by Puntius chola, Pethia conchonius and Barilius shacra in Behni and Karnal stream while the cascade habitats are colonized by Labeo pangusia and Garra gotyla gotyla in Behni stream. Dammed pools, backwater pools and deep pool edges with bedrock substratum are the highly preferred habitats for Botia birdi, Botia lohachata, Tor tor and Cirrhinus reba as found in Basoli stream and main reservoir. Barilius bendelisis and Acanthocobitis botia are abundant in the riffle-type of habitats in the Basoli, Behni and Karnal stream where the substrata have been found to be mainly dominated by small boulders and cobbles. Among the cyprinids, Tor tor and Tor piutitora are confined to large deep pools in Basoli stream and main reservoir. Nevertheless, species like Crossocheilus latius latius, Garra gotyla gotyla and Glyptothorax punjabensis have been recorded from the cascade to riffle regions in the upper gradient zones of rheophilic streams.

In India, the introduction of exotic fish species into Dal lake and Loktak lake has been reported to affect the population of indigenous fish species. The population of native catla and mahseer were depleted considerably in Gobind Sagar reservoir after the introduction of Silver Carp. The freshwater aquatic biodiversity is depleting alarmingly due to introduction of exotic species and other anthropogenic activities (Menon 1979; Molur and Walker 1998; Kumar 2000). The indiscriminate transfers of exotic fishes have brought about a wide array of problems including extirpation of indigenous species. During the present course of work four exotic carps i.e. Cyprinus carpio, Ctenopharyngodon idellus, Hypophthalmichthys molitrix and Hypophthalmichthys nobilis have been reported from the Ranjit Sagar Wetland. It is important to note that these exotic carps are voracious feeders as well as breeders and can pose serious threats to native fauna 
in future if proper management and research activities will not be initiated about their control (Kumar 2000).

Though this wetland and its adjoining streams were highly productive, but some anthropogenic activities like rampant removal of big and small boulders from the stream bed, mining of sand and gravel by builders or constructor and siltation posing a big threat to the various fish species. Besides, these serious threats, discharge of sewage water and poaching of fishes have also been consider as potential threats to the fish diversity which need to be curbed for their sustainability.

\section{Conclusion}

During the study period 43 species have been reported from the Ranjit Sagar Wetland due to availability of variety of habitats. Out of all 43 fish species 13 fish species come under threatened categories of Red List of IUCN. There is a dire need to start conservation and management practices for their sustainability in future. If appropriate steps towards their conservation will not be taken of now, then the day is not far away when these fish species will slip towards extinction. This wetland also supports four exotic carps and these are voracious feeders as well as breeders and can pose serious threats to native fauna in future. Proper management and research activities should be initiated for the conservation of valuable fish fauna of this wetland.

\section{Acknowledgements}

We express our special thanks to the Ministry of Environment, Forests and Climate Change, Government of India for providing funds to conduct this study and authors are also thankful to the Head, Department of Zoology and Environment Sciences, Punjabi University Patiala for providing necessary laboratory facilities to carry out the work.

\section{REFERENCES}

Abbasi SA (1997) Wetlands of India-ecology and threats: the Wetlands of Kerla. New Delhi: Discovery Publication House, pp $1-151$.

Armantrout NB (1990) Conservation in developing countries. In: Daniel JC, Serrao JS (eds) Problem and prospects, proceedings of the centenary seminar on the Bombay National History Society.

Arunachalam M (2000) Assemblage structure of stream fishes in the Western Ghats (India). Hydrobiologia 430: 1-31.

Ayappan S, Birdar SR (2004) Enhancing Global Competition. Survey of Indian Agriculture (The Hindu).

Baker JA, Olem H, Creager CS, Marcus MD, Parkhurst BR (1993) Fish and fisheries management in lakes and reservoirs. EPA
841-R-93-002. Washington DC: Terrene Institute and US Environmental Protection Agency.

Brraich OS, Ladhar SS (2005) Fish Biodiversity in Wetlands of Punjab - A Field Guide. Chandigarh: Punjab State Council for Science and Technology, pp 1-102.

Brraich OS, Ladhar SS, Johal MS (2003) New fish record from Harike wetland, Punjab-Ramsar Site. Punjab Fisheries Bulletin XXIII: pp 35-36.

Daniels RJR (2000) Project lifescience 6. Freshwater fishes: Cat-fishes. Resonance 5: 95-107.

Day F (1888) Fishes of India. Supplement, October, 1888. William Dawson, London.

Dua A, Chander P (1999) Fish and bird diversity at Harike wetland - A Ramsar site. In: International Conference on Tropical Aquatic Ecosystems. Nainital, India: Health, Management and Conservation.

Gharaei A (2010) Morphometric and meristic studies of Snow Trout Schizothorax zarudnyi (Nikolskii 1897) as A Threatened Endemic Fish. World J Fish Mar Sci 4: 426-429.

Gharaei A, Rahdari A, Ghaffari M (2011) Induced spawning of Schizothorax zarudnyi (Cyprinidae) by using synthetic hormones (Ovaprim and HCG). World J Fish Mar Sci 3: 518-522.

Goswami TK, Goswami MM (2006) Icthyofaunal diversity and catch statistics of Jamlai wetland in Kamrup district of Assam, India. J Inland Fish Soc India 38: 38-44.

Hawkins CP, Kershner JF, Bisson PA, Bryant MD, Decker LM, Gregory SV, McCullough DA, Overton CK, Reeves GH, Steedman RJ, Young MK (1993) A hierarchial approach to classifying stream habitat features. Fisheries 18: 3-12.

Jagatheeswari J, Kishore B, Rajasekhar PS (2016) Fish diversity and conservation aspects in an aquatic ecosystem, Kondakarla fresh water lake, Visakhapatnam, Andhra Pradesh, India. Int J Fish Aquat Stud 4: 20-23.

Jayaram KC (2010) The freshwater fishes of the Indian region (2nded.). K.C. Jayaram (ed.). New Delhi, India: Narendra Publishing House.

Johal MS, Tandon KK (1979) Monograph on the fishes of Reorganized Punjab, Part I. Punjab Fisheries Bulletin 3: 1-44.

Johal MS, Tandon KK (1980) Monograph on the fishes of Reorganized Punjab, Part II. Punjab Fisheries Bulletin 4: 39-70.

Johal MS, Tandon KK, Tyor AK, Rawal YK (2002) Fish diversity in different habitats in the streams of lower, middle Western Himalayas. Pol J Ecol 50: 45-56.

Kar D, Sen N (2007) Systematic list and distribution of fishes in Mizoram, Tripura and Barak drainage of northeastern India. Zoos Print J 22: 2599-2607.

Kar D, Dey SC, Datta NC (2003) Welfare Biology in the New Millenium. Allied Publishers Pvt. Ltd., Bangalore.

Kumar AB (2000) Exotic fishes and freshwater fish diversity. Zoos Print J 15: 363-367.

Menon AGK (1979) Conservation of the Ichthyofauna of India. In: Conservation and Management of Inland Capture Fisheries Resources of India. Jhingran AG, Sugnan VV (eds), pp 25-33.

Molur S, Walker S (1998) Conservation Assessment and Management Plan (CAMP) Workshop Report on Freshwater Fishes of India. Coimbatore, India: Zoo Outreach Organisation, CBSG, pp 1-156.

Plafkin JL, Barbour MT, Porter KD, Gross SK, Hughes RM (1989) Rapid Bioassessment protocols for use in streams and rivers: benthic macroinvertebrates and fish. United States Environmental Protection Agency, Washington, DC 20460.

Rosenzweig ML (1995) Species diversity in space and time. Cambridge: Cambridge University Press. 
Rosgen DL (1996) Applied River Morphology, Wildland Hydrology Books, Pagosa Springs, Colorado: Ft. Collins, CO.

Ross ST (1986) Resource partitioning in fish assemblages: a review of field guide studies. Copiea 352-388.

Sharma KK, Nitasha, Sarbiet K (2007) Some limnological investigations in Banganga stream Koba. Jammu and Kashmir State. J Aquat Biol 22: 105-109.

Sikoki FD, Otobotekere AJT (1999) Fisheries. The Land People of Bayelsa State Central Niger Delta, Port Harcourt, Nigeria.

Siligato S, Bohmer J (2001) Using indicators of fish health at multiple levels of biological organization to assess effects of stream pollution in southwest Germany. J Aquat Ecosyst Stress Recovery 8: $371-386$.

Tiwari RN (2011) Assessment of groundwater quality and pollution potential of Jawa Block Rewa District, Madhya Pradesh, India. In: Proceedings of the International Academy of Ecology and Environmental Science 1: 202-212.

Vijaylaxmi CM, Rajshekhar, Vijaykumar K (2010) Freshwater fishes distribution and diversity status of Mullameri River, a minor tributary of Bheema River of Gulbarga District, Karnataka. Int J Syst Biol 2: 1-9. 